\title{
The effect of apatinib in the treatment of metastatic renal cell carcinoma: a case report and review of the literature
}

This article was published in the following Dove Press journal:

International Medical Case Reports Journal

4 July 2017

Number of times this article has been viewed

Jinling $\mathrm{Bi}$

Haiyuan Liu

Yong Huang

Department of Oncology, The Second People's Hospital of He Fei, He Fei, An Hui, People's Republic of China
Correspondence: Yong Huang Department of Oncology, The Second People's Hospital of He Fei, No 246 He Ping Road, He Fei, An Hui 23000I, People's Republic of China

Tel +8655I 62965370

Email huangyong1965@126.com

\begin{abstract}
The aim of this study was to explore the effect of apatinib in the treatment of metastatic renal cell carcinoma ( $\mathrm{mRCC}$ ) and related adverse events. A case of $\mathrm{mRCC}$ was reported which recurred after surgery and roferon treatment. The patient was treated with apatinib at a dose of $500 \mathrm{mg}$ orally, twice daily, 28 days/cycle. The metastatic lesions improved based on computed tomography after apatinib administration in the fourth and eighth month. The progression-free survival of the patient had increased almost to 8 months. The patient showed a good tolerance with only an adverse effect of mild-to-moderate hand-foot syndrome which was managed well. Apatinib is an option for mRCC after previous treatment. However, more and larger trials are still needed. Keywords: metastatic renal cell carcinoma, apatinib, sunitinib, sorafenib, vascular endothelial growth factor receptor
\end{abstract}

\section{Introduction}

The incidence of renal cell carcinoma (RCC) has continuously increased worldwide, and almost one-third of patients with RCC have been confirmed as having metastatic disease at the initial diagnosis. Generally, the prognosis of metastatic RCC (mRCC) is poor even when surgery is performed due to insensitivity to regular chemotherapy and radiotherapy agents. Pathologically, clear cell carcinoma is the most common type of RCC. Recently, in a study focusing on a Chinese population, it was found that an interleukin-6 gene polymorphism may be predictive for susceptibility and prognosis of RCC. ${ }^{1}$ Some studies have shown the involvement of different pathways during the development of RCC. The RhoA-ROCK pathway can be inhibited by PARG1, a new RCC antigen, which leads to mRCC and further poor survival. ${ }^{2}$ Meanwhile, the Wnt/ $\beta$-catenin signaling pathway also plays an important role in the progression of RCC. ${ }^{3}$ Some special factors have been identified as prognostic parameters for RCC including neutrophil to lymphocyte ratio and tumoral CCR7. 4,5

Currently, targeted therapy is recommended to treat locally advanced RCC and mRCC before surgery due to safety, feasibility and fewer complications. ${ }^{6}$ Clinically, apatinib, an oral antiangiogenic agent, is widely used during the treatment of different tumors. In particular, apatinib is the most common targeted agent aiming to improve progression-free survival (PFS) and overall survival (OS) of patients with advanced gastric cancer (AGC) and metastatic gastric cancer (mGC) after failure of chemotherapy. ${ }^{7}$ Nevertheless, few publications related to the effect and safety of apatinib in treating $\mathrm{mRCC}$ have been found. Herein, a case of $\mathrm{mRCC}$ managed with apatinib is presented. 


\section{Case presentation}

A 62-year-old male patient was admitted to our hospital on May 10, 2016, complaining of a fist-like mass on his back complicated with hemoptysis for 10 days. The patient had been diagnosed with RCC and treated by nephrectomy of the left kidney 15 years previously (May 26, 1999). The pathology test after surgery showed clear cell carcinoma of the left kidney with dimensions of $6 \mathrm{~cm} \times 6 \mathrm{~cm} \times 4 \mathrm{~cm}$, and no metastasis was found in a total of seven tested lymph nodes or in the resection margin of the ureter. The patient had been treated with $9 \mathrm{MIU} /$ week roferon for 3 consecutive months after discharge. During the follow-up, no discomfort had been reported and physical examination of the patient revealed that normal findings were maintained. However, the patient was admitted again 2 years ago (August 21, 2014) complaining of hemoptysis which had persisted for 1 week. A chest computed tomography (CT) scan showed that there was an isolated mass in the lower lobe of the right lung. Positron emission tomography (PET) showed that a peripheral mass was located in the lower lobe of the right lung without any other metastasis, and the susceptibility to cancer was not excluded in combination with a smoking history of more than 40 years. Video-assisted pulmonary wedge resection was performed on August 26, 2014. However, the pathology test indicated lung metastasis of renal clear cell carcinoma (lower lobe of right lung) with dimensions of $4 \mathrm{~cm} \times 4 \mathrm{~cm} \times$ $2 \mathrm{~cm}$. One metastatic lymph node was identified among six tested subcarinal nodes, and no metastasis was found in a total of 14 mediastinal nodes. Immunolabeling revealed the tumor to be CD10+, CK8+, Vim+, EMA+, CK+, CD34-, inhibin-, and Ki-67+ (10\%), TTF-1-, Napsin-A- and p63(Figure 1). Written informed consent for the publication of the patient's clinical details and accompanying images was obtained from the patient.

No special treatment was given after discharge. Before the third admission, the patient's condition was stable without any complaints during the 2-year follow-up. A CT scan (April $29,2016)$ showed the presence of multiple intrapulmonary

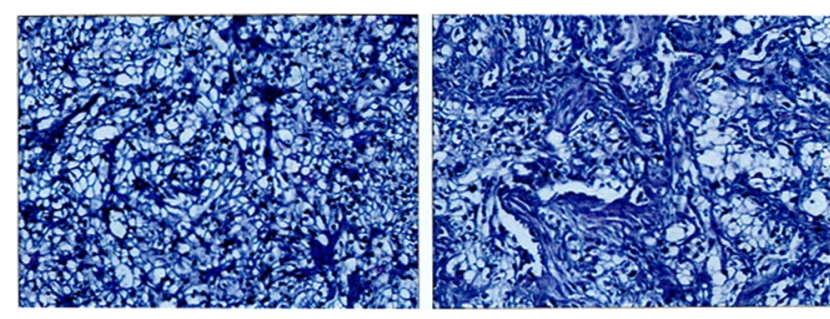

Figure I Pathology test indicated lung metastasis of renal clear cell carcinoma. Notes: Immunolabeling revealed the tumor to be CDI0+, CK8+, Vim+, EMA+, CK+, CD34-, inhibin-, and Ki-67+ (10\%), TTF-I-, Napsin-A- and p63-. Magnification $\times 400$. metastatic lesions located mainly within the middle and lower lobes of the right lung and a larger lesion with dimensions of $3.6 \mathrm{~cm} \times 6.3 \mathrm{~cm}$ located within the basal segment of the lower lobe extending partially outside the thoracic cavity. Osteolytic destruction was found in adjacent ribs. No swollen mediastinal lymph nodes were found (Figure 2). Based on these findings together with past medical history, the patient was diagnosed with lung metastasis of renal clear cell carcinoma, TxN1M1, IV stage.

An individual treatment strategy was prepared. Traditionally, sunitinib and sorafenib have been recommended as second- or third-line treatment for the treatment of mRCC; however, the patient refused these two agents due to poor financial support. Hence, apatinib (Hengrui Pharmaceutical Co., Ltd., Lian Yun Gang, Jiang Su, China) was used to manage the disease. After all contraindications were excluded, based on his individual condition observed in clinical practice, the patient took apatinib orally at a dose of $500 \mathrm{mg} /$ day, starting on May 3, 2016 (28 days/cycle). A CT scan was performed every 4 months to evaluate the effect. Routine tests of blood and biochemical function as well as assessment of adverse events were performed every 2 weeks. Approximately 2 weeks later, the mass on the patient's back was observably smaller and hemoptysis had cleared up; however, the patient also complained of pain in the hands and feet. During physical examination, his hands and feet were found to be red and a little swollen but without peeling or blistering. Mild hand-foot syndrome was considered and regular preventive treatment was recommended. Approximately 4 weeks later, the patient complained that the pain was not relieved as expected. Oral celecoxib $100 \mathrm{mg}$ twice daily was given for the intensity of pain management. The patient reported dramatic pain relief following analgesic therapy. Regular routine test results were stable.
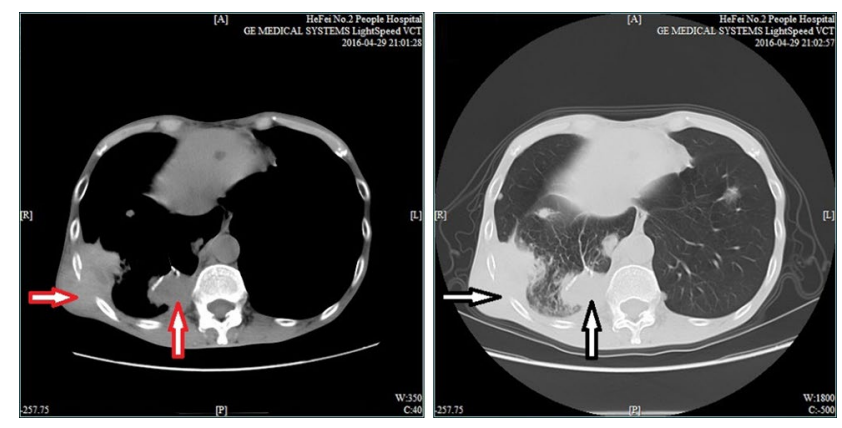

Figure 2 Chest CT scan indicates a large lesion with dimensions of $3.6 \mathrm{~cm} \times 6.3 \mathrm{~cm}$ (red and white vertical arrows) located within the basal segment of the lower lobe and extending out of the thoracic cavity, partially complicated with osteolytic destruction (red and white horizontal arrows).

Note: Lung window, left; mediastinum window, right.

Abbreviation: CT, computed tomography. 
The patient was rechecked by chest CT scan 4 months later. The results (August 27, 2016) revealed multiple intrapulmonary metastatic lesions located mainly within the middle and lower lobes of the right lung and a larger lesion with dimensions of $2.7 \mathrm{~cm} \times 1.8 \mathrm{~cm}$ located within the basal segment of the lower lobe and partially extending out of the thoracic cavity. Worse osteolytic destruction was observed in adjacent ribs (Figure 3). The effect of apatinib was evaluated as a partial response (PR). Therefore, apatinib was continued as planned and zoledronic acid was used for the inhibition of bone destruction. Results of regular routine tests including blood, urine and stool remained stable.

A third CT evaluation was performed on December 24, 2016. The chest CT scan showed the presence of multiple intrapulmonary metastatic lesions located mainly within the middle and lower lobes of the right lung and a larger lesion with dimensions of $2.7 \mathrm{~cm} \times 1.9 \mathrm{~cm}$ located within the basal segment of the lower lobe and partially extending out of the thoracic cavity. Improved osteolytic destruction was observed in the adjacent ribs (Figure 4). Meanwhile, abdominal and
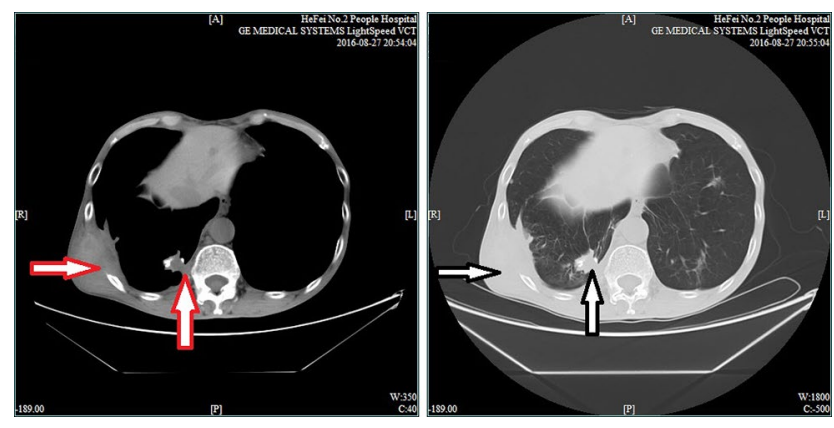

Figure 3 Chest CT scan indicates a larger lesion with dimensions of $2.7 \mathrm{~cm} \times 1.8 \mathrm{~cm}$ (red and white vertical arrows) located within the basal segment of the lower lobe and extending out of the thoracic cavity, partially complicated with worse osteolytic destruction (red and white horizontal arrows).

Note: Lung window, left; mediastinum window, right.

Abbreviation: CT, computed tomography.
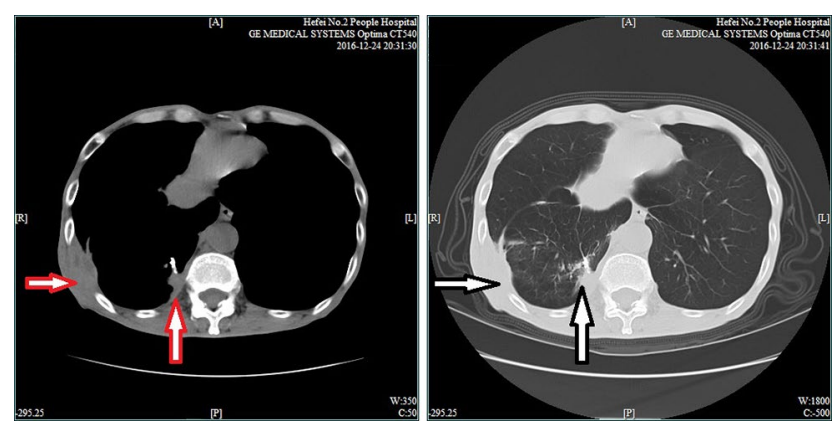

Figure 4 Chest CT scan indicates a larger lesion with dimensions of $2.7 \mathrm{~cm} \times$ $1.8 \mathrm{~cm}$ (red and white vertical arrows) located within the basal segment of the lower lobe and partially extending out of the thoracic cavity, complicated with improved osteolytic destruction (red and white horizontal arrows).

Note: Lung window, left; mediastinum window, right.

Abbreviation: CT, computed tomography. pelvic CT scans were also performed, which indicated that no metastases were found, similar to the results of previous PET. The evaluation was still PR. To date, the patient has achieved a PFS of almost 8 months. During the apatinib cycles, mild-to-moderate hand-foot syndrome has developed and has been treated with appropriate agents.

\section{Discussion}

Generally, the approved targeted agents for mRCC are sunitinib and sorafenib. Sunitinib is considered as a small molecule inhibitor of receptor TKI including platelet-derived growth factor receptor (PDGFR) and vascular endothelial growth factor receptor (VEGFR). Therefore, besides the inhibition of PDGFR and VEGFR, the mechanism of sorafenib also activates the inhibition of RAF/MEK/ERK signaling. In a study in a Latin-American population, sunitinib has been confirmed to be safe and effective even with worse prognosis. The median PFS (mPFS) and OS were 12.1 and 16.9 months, respectively, while neutropenia, thrombocytopenia and anemia were common adverse hematologic events. ${ }^{7}$ Rovithi et $\mathrm{al}^{8}$ indicated that the optimal treatment with sunitinib was based on a pulsatile, high-dose strategy, which led to an antitumor effect. Chang et $\mathrm{al}^{9}$ suggested that among patients with $\mathrm{RCC}$, the genotype of cyclooxygenase 2 (COX2) could be used as a novel marker for the diagnosis of RCC. In 14 patients with inoperable $\mathrm{mRCC}$, neoadjuvant sunitinib therapy showed a satisfactory response rate although a small number are operable after treatment. ${ }^{10}$ It has been indicated that among Chinese patients with $\mathrm{mRCC}$, both sorafenib and sunitinib are effective agents. The median OS of patients treated with the two agents is similar ( 24.0 vs 24.0 months; $P=0.298$ ), while the mPFS with sorafenib is higher compared with sunitinib (11.1 vs 10.0 months; $P=0.028$ ). In addition, sorafenib is recommended to elderly patients aged over 65 years. ${ }^{11}$ A study in Tsc $2^{ \pm}$mice by Yang et al ${ }^{12}$ indicated that everolimus used as monotherapy or combined with sorafenib is effective in controlling tumor size, based on the inhibitory mechanism of mTORC1 and the mitogen-activated protein kinase (MAPK) pathway. Nevertheless, there is also a difference between sorafenib and sunitinib. Ma et $\mathrm{al}^{13}$ indicated that there is a correlation between these two agents and the expression levels of hypoxia-inducible factor 2, alpha subunit (HIF-2 $\alpha$ ), CD31, carbonic anhydrase IX (CAIX), vascular endothelial growth factor receptor 1 (VEGFR-1) and platelet-derived growth factor beta polypeptide (PDGFRB), and interestingly the opposite trend is also found for sorafenib and sunitinib. In a single-center retrospective study, a total of 169 patients receiving sorafenib (400 mg, twice daily, 4 weeks/cycle) and 
165 patients receiving sunitinib (50 mg/day, 6 weeks/cycle) were enrolled. The PFS and OS of sorafenib and sunitinib were similar (9.0 vs 11.0 months, respectively; 28.0 vs 28.0 months, respectively). For vascular endothelial growth factor-TKI (VEGF-TKI)-naive patients with $\mathrm{mRCC}$, the effect of sorafenib and sunitinib was also comparable (9.0 vs 11.0 months, respectively). However, sorafenib was found to be superior in subsets such as non-clear cell carcinoma, with Karnofsky Performance Status (KPS) $<90 .{ }^{14}$ Bourlon et $\mathrm{al}^{15}$ found that compared with sorafenib, the incidence of macrocytosis was significantly higher during sunitinib treatment. Moreover, organ atrophy has been demonstrated during treatment with either sorafenib or sunitinib. The atrophy of the spleen, thyroid gland and pancreas was significant as assessed by CT scan within 1 year of treatment. ${ }^{16}$

Other targeted therapies for $\mathrm{mRCC}$ are potentially significant. For clear-cell mRCC, bevacizumab and pazopanib, as first-line treatment, can significantly improve PFS. On the other hand, temsirolimus is better for improving poor prognosis of clear cell and non-clear cell mRCC. ${ }^{17}$

For mRCC, the pathways of VEGFR and mammalian target of rapamycin (mTOR) are considered to be the main mechanisms of carcinogenesis. It has been proposed that dysfunction of von Hippel-Lindau (VHL) is the key factor in dysregulation of VEGF signaling which leads to overexpression of VEGF protein and increased tumor angiogenesis. ${ }^{18}$ Recently, it has been proposed that combination therapy with TKIs of VEGFR, such as sorafenib and sunitinib and the mTOR inhibitors, vatalanib and everolimus, is effective and tolerable for patients suffering from mRCC. ${ }^{19}$ Terakawa et $\mathrm{a}^{20}$ suggested that VEGFR-2 is the most significant factor in PFS of mRCC in a study involving a total of 40 patients with $\mathrm{mRCC}$ after radical nephrectomy. It has been shown in a registry-based analysis that anti-VEGFR agents are effective in improving the prognosis of mRCC. ${ }^{21}$ Apatinib, as a small-molecule multi-targeted TKI of VEGFR-2, potentially interacts with CYP1A2, CYPAD4 and CYP3A1 of the cytochrome $\mathrm{P} 450$ family in vivo. ${ }^{22}$ Apatinib, which inhibits VEGFR-2, may further reduce endothelial cell migration and proliferation as well as decrease tumor microvascular density. ${ }^{23}$ Peng et $\mathrm{al}^{24}$ have found that among patients with intrahepatic cholangiocarcinoma (ICC), apatinib plays an important role in inhibiting VEGF-VEGFR-2-PI3K-AKT signaling and inducing apoptosis of ICC cells.

It has been confirmed that VEGF plays a key role in the development of carcinoma and that VEGFRs function as regulators of this process. ${ }^{25}$ Apatinib, a small-molecule antiangiogenic agent, shows an inhibitory effect on VEGFR-2 and a moderate inhibitory effect on c-Kit and c-Src TKIs. ${ }^{26}$ Apatinib exerts its inhibitory effect by binding to VEGFR-2 and inhibiting phosphorylation of extracellular signalregulated kinase (ERK). ${ }^{27}$ Currently, apatinib is used as monotherapy or combination therapy with other chemotherapy agents against various solid tumors and shows better tolerance. The most common adverse events of apatinib are hypertension, proteinuria and hand-foot syndrome.

Apatinib has been assessed in a randomized Phase II trial focusing on AGC or $\mathrm{mGC}$ with high expression of VEGFR. ${ }^{28}$ Placebo, apatinib $850 \mathrm{mg} /$ day and apatinib $425 \mathrm{mg}$ twice daily were the three study groups. The PFS and OS of the two apatinib groups were significant better compared with the placebo group; however, there was no significant difference between the two groups, which meant that apatinib improved PFS and OS for patients with AGC or mGC after failure of chemotherapy. Recently, Peng et $\mathrm{al}^{24}$ demonstrated that the development of ICC relied on the interaction between VEGF and VEGFR-2. Consequently, targeting of the VEGF signaling pathway by apatinib may be recommended as treatment for ICC. Treatment of metastatic non-triple-negative breast cancer (TNBC) is challenging due to drug resistance. In a Phase IIb study, a total of 59 patients with TNBC received apatinib at a dose of $500 \mathrm{mg} /$ day. The mean PFS and mean OS were 3.3 and 10.6 months, respectively, and the most common adverse events were thrombocytopenia, leukopenia, neutropenia and anemia. ${ }^{29}$ Song et $\mathrm{al}^{30}$ reported that mPFS and OS of patients with non-small cell lung cancer (NSCLC) treated with apatinib was 4.2 and 6 months, respectively, in a trial involving 42 patients with NSCLC who received apatinib at a dose of $500 \mathrm{mg} /$ day.

In the case presented in this article, the patient refused sorafenib and sunitinib because of poor financial support. However, the patient also had a strong desire to continue treatment after failure of previous treatments including interferon- $\gamma($ IFN- $\gamma)$. Based on individual condition and clinical effects as well as the benefits of apatinib, this drug was selected for the patient. We report that it is a reasonable and feasible therapy when given at a level of $500 \mathrm{mg}$ orally twice per day. Mild-to-moderate hand-foot syndrome was observed but was well managed.

\section{Conclusion}

In this case, apatinib was used to treat $\mathrm{mRCC}$ and, so far, the effect is good. However, the effect of apatinib on different tumors is variable due to the different mechanisms involved. Hence, more and larger trials are needed in future to further explore the effects. Moreover, the indications concerning 
monotherapy and combination therapy are also identified during clinical practice.

\section{Disclosure}

The authors report no conflicts of interest in this work.

\section{References}

1. Liu Z, Wang Z, Xiao Y, Lu Y, Lu Y. Association between the interleukin-6 gene polymorphisms and renal cancer risk. Immunol Lett. 2015;164(2):125-128.

2. Miyazaki J, Ito K, Fujita T, et al. Progression of human renal cell carcinoma via inhibition of RhoA-ROCK axis by PARG1. Transl Oncol. 2017;10(2):142-152.

3. Liu Z, Liu XW, Liu SA, Lv JJ, Fu Q. Clinical significance of changes of expression of the Wnt/ $\beta$-catenin signaling pathway in renal clear cell carcinoma. Eur Rev Med Pharmacol Sci. 2016;20(23):4840-4845.

4. Bazzi WM, Tin AL, Sjoberg DD, Bernstein M, Russo P. The prognostic utility of preoperative neutrophil-to-lymphocyte ratio in localized clear cell renal cell carcinoma. Can J Urol. 2016;23(1):8151-8154.

5. Xia Y, Liu L, Xiong Y, et al. Prognostic value of CC-chemokine receptor seven expression in patients with metastatic renal cell carcinoma treated with tyrosine kinase inhibitor. BMC Cancer. 2017;17(1):70-80.

6. Borregales LD, Adibi M, Thomas AZ, Wood CG, Karam JA. The role of neoadjuvant therapy in the management of locally advanced renal cell carcinoma. Ther Adv Urol. 2016;8(2):130-141.

7. Barrios CH, Herchenhorn D, Chacón M, Cabrera-Galeana P, Sajben P, Zhang K. Safety and efficacy of sunitinib in patients from Latin America: subanalysis of an expanded access trial in metastatic renal cell carcinoma. Onco Targets Ther. 2016;9(23):5839-5845.

8. Rovithi M, de Haas RR, Honeywell RJ, et al. Alternative scheduling of pulsatile, high dose sunitinib efficiently suppresses tumor growth. J Exp Clin Cancer Res. 2016;35(1):138-149.

9. Chang WS, Liao CH, Miao CE, et al. The role of functional polymorphisms of cyclooxygenase 2 in renal cell carcinoma. Anticancer Res. 2014;34(10):5481-5486.

10. Kim SH, Seo SI, Lee HM, et al. A prospective multicenter trial of the efficacy and tolerability of neoadjuvant sunitinib for inoperable metastatic renal cell carcinoma. J Korean Med Sci. 2016;31(12):1983-1988

11. Zhang HL, Sheng XN, Li XS, et al. Sorafenib versus sunitinib as firstline treatment agents in Chinese patients with metastatic renal cell carcinoma: the largest multicenter retrospective analysis of survival and prognostic factors. BMC Cancer. 2017;17(1):16-26.

12. Yang J, Samsel PA, Narov K, et al. Combination of everolimus with sorafenib for solid renal tumors in Tsc $2 \pm$ mice is superior to everolimus alone. Neoplasia. 2017;19(2):112-120.

13. Ma X, Wang L, Li HZ, et al. Predictive immunohistochemical markers related to drug selection for patients treated with sunitinib or sorafenib for metastatic renal cell cancer. Sci Rep. 2016;6:30886.

14. Sheng XN, Chi ZH, Cui CL, et al. Efficacy and safety of sorafenib versus sunitinib as first-line treatment in patients with metastatic renal cell carcinoma: largest single-center retrospective analysis. Oncotarget. 2016;19(7):27044-27054.
15. Bourlon MT, Gao DX, Trigero S, et al. Clinical significance of sunitinibassociated macrocytosis in metastatic renal cell carcinoma. Cancer Med. 2016;5(12):3386-3393.

16. Takahashi H, Nasu K, Minami M, et al. Organ atrophy induced by sorafenib and sunitinib-quantitative computed tomography (CT) evaluation of the pancreas, thyroid gland and spleen. Pol J Radiol. 2016;81(22):557-565.

17. Afriansyah A, Hamid AR, Mochtar CA, Umbas R. Targeted therapy for metastatic renal cell carcinoma. Acta Med Indones. 2016;48(4): 335-347.

18. Rini BI, Escudier B, Tomczak P, et al. Comparative effectiveness of axitinib versus sorafenib in advanced renal cell carcinoma (AXIS): a randomised phase 3 trial. Lancet. 2011;378(9807):1931-1939.

19. Rhonda LB, Patrick H, Patricia AC, et al. A phase Ib study of combined VEGFR and $m$ TOR inhibition with vatalanib and everolimus in patients with advanced renal cell carcinoma. Clin Genitourin Cancer. 2014;4(12): 241-250.

20. Terakawa T, Miyake H, Kusuda Y, Fujisawa M. Expression level of vascular endothelial growth factor receptor-2 in radical nephrectomy specimens as a prognostic predictor in patients with metastatic renal cell carcinoma treated with sunitinib. Urol Oncol. 2013;31(4): 493-498.

21. Tomas B, Zbynek B, Alexandr P, et al. Outcomes for patients with metastatic renal cell carcinoma achieving a complete response on targeted therapy: a registry-based analysis. Eur Urol. 2016;70(3):469-475.

22. Zhou YF, Wang SH, Ding T, et al. Evaluation of the effect of apatinib (YN968D1) on cytochrome P450 enzymes with cocktail probe drugs in rats by UPLC-MS/MS. J Chromatogr B Analyt Technol Biomed Life Sci. 2014;15(973):68-75.

23. Li J, Zhao XM, Chen L, et al. Safety and pharmacokinetics of novel selective vascular endothelial growth factor receptor-2 inhibitor YN968D1 in patients with advanced malignancies. BMC Cancer. 2010;10:529.

24. Peng H, Zhang Q, Li J, et al. Apatinib inhibits VEGF signaling and promotes apoptosis in intrahepatic cholangiocarcinoma. Oncotarget. 2016;7(13):17220-17229.

25. Zhang HJ. Apatinib for molecular targeted therapy in tumor. Drug Des Devel Ther. 2015;9(13):6075-6081.

26. Tian S, Quan H, Xie C, et al. YN968D1 is a novel and selective inhibitor of vascular endothelial growth factor receptor-2 tyrosine kinase with potent activity in vitro and in vivo. Cancer Sci. 2011;102(7): 1374-1380.

27. Scott AJ, Messersmith WA, Jimeno A. Apatinib: a promising oral antiangiogenic agent in the treatment of multiple solid tumors. Drugs Today (Barc). 2015;51(4):223-239.

28. Li J, Qin S, Xu J, et al. Apatinib for chemotherapy-refractory advanced metastatic gastric cancer: results from a randomized, placebo-controlled, parallel-arm, phase II trial. J Clin Oncol. 2013;31(26):3219-3225.

29. Hu X, Zhang J, Xu B, et al. Multicenter phase II study of apatinib, a novel VEGFR inhibitor in heavily pretreated patients with metastatic triple-negative breast cancer. Int J Cancer. 2014;135(8):1961-1969.

30. Song ZB, Yu XM, Lou GY, et al. Salvage treatment with apatinib for advanced non-small-cell lung cancer. Onco Targets Ther. 2017;23(10): $1821-1825$.
International Medical Case Reports Journal

\section{Publish your work in this journal}

The International Medical Case Reports Journal is an international, peer-reviewed open-access journal publishing original case reports from all medical specialties. Previously unpublished medical posters are also accepted relating to any area of clinical or preclinical science. Submissions should not normally exceed 2,000 words or

\section{Dovepress}

4 published pages including figures, diagrams and references. The manuscript management system is completely online and includes a very quick and fair peer-review system, which is all easy to use. Visit $\mathrm{http}: / /$ www.dovepress.com/testimonials.php to read real quotes from published authors. 